\title{
KM System Evaluation using Four dimensional Metric Model, Database and RESTful Resources
}

\author{
D.Venkata Subramanian ${ }^{1}$, Angelina Geetha ${ }^{1}$, K.M. Mehata ${ }^{1}$, \\ K.Mohammed Hussain ${ }^{2}$ \\ ${ }^{1}$ Department of Computer Science and Engineering \\ ${ }^{2}$ Department of Computer Applications \\ B.S. Abdur Rahman University, Chennai, India
}

\begin{abstract}
A Knowledge Management (KM) System plays a crucial role in every industry as well as in Higher Learning Institutions. A RESTful resource is anything that is addressable over the Web. The resources can be accessed and transferred between clients and server. The resources can be accessed and transferred between clients and servers. Based on our earlier research works, we have developed a comprehensive KM System framework, evaluation method, mult-dimensional metric model and useful metrics which are helpful to assess any given knowledge management system. In this proposed work, we first describe the actual implementation steps for building the KM System metric database using the multi-dimensional metric model. Secondly we describe the approaches for designing a multi-dimensional Restful Resources and Web Services using the mutli-dimensional metric model and demonstrate how the KM system can be ranked and rated for its effectiveness using WAM and RESTful Resources.
\end{abstract}

\section{KEYWORDS}

Knowledge Management Systems (KMS), Metrics Model, KM Metrics, Multi-dimensional model, Evaluation, Metrics Database, Ranking, Rating, Weighted Average Mean(WAM), RESTful Resources

\section{INTRODUCTION}

Knowledge Management (KM) provides an innovative methodology for creating and modifying to promote knowledge creation and sharing. Many companies and institutions are working towards building an effective KMS as well as using collaborative tools for increasing the knowledge sharing with their knowledge workers. One of the key challenges with the KMS's is evaluation of the knowledge available within the organization and assessing the capabilities and effectiveness of the KMS infrastructure [1]. Assessing the worth of the information and the infrastructure within the organization is a crucial step if an organization wants to change the knowledge capture methods and rewarding system for employees who have contributed the best knowledge asset. Considering the intangible nature of the knowledge asset, complexity and dynamics of building the KM System infrastructure, one of an approach is, to determine the strengths and weakness through metrics. Metrics can be collected through an evaluation methodology such as Goal Question Metrics (GQM), Weighted Balanced Score Card and/or using Hybrid methodology [2]. Based on many research works, it has been identified that there are no proven reliable metric model and metric database to estimate and report the worth of the knowledge being shared and also the worth of KM Systems. This paper first describes a comprehensive KM System framework, Metrics and 
Measurement Process which are critical for conducting an evaluation exercise on a KM System. Secondly this paper describes the process of building metric database using the multi-dimensional metric model for capturing the measures and metrics which are collected through evaluation methods and illustrate how to apply WAM Method to rank and rate the effectiveness of a given KM system. This paper finally describes the process of building RESTful Resources and Web Services for evaluating KM Systems.

\section{KMS FRAMEWORK}

Knowledge management $(\mathrm{KM})$ system is a collective term that is used to describe the creation of knowledge repositories, respective interface components, improvement of knowledge access and sharing as well as communication through collaboration, enhancing the knowledge environment and managing knowledge as an asset for an organization. Considering the fundamental capabilities of KMS and typical KMS infrastructure topology, we have identified a suitable KMS framework [2] which is mentioned in the below figure (Figure 1). This framework represents all the components which make up the KMS for industries as well as higher learning institutions and in particular focused on the needed quality factors to develop a measurement model based which are helpful for measuring the effectiveness of KMS.

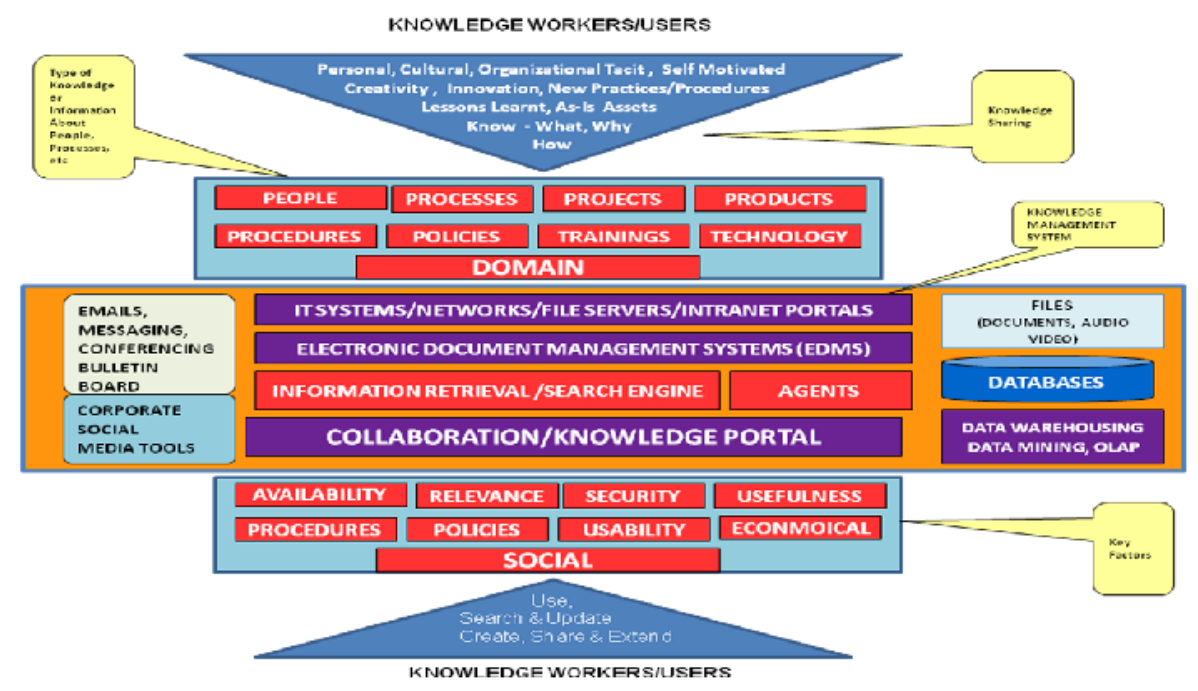

Figure 1: KMS Framework

\section{KM SYSTEM EVALUATION PROCESS}

\subsection{Metrics}

A useful metric should be measurable, independent, accountable \& precise. As much as possible, the KM measures should be related to, or the same as, existing measures in the organization that are used to monitor the success of performing mission objectives. For evaluating the capability of the KM system, the metric must indicate the capabilities of the KMS. 


\subsection{Evaluation}

An experiment process deliberately imposes a treatment on a group of objects or subjects in the interest of observing the response. As illustrated in the Figure 2, the basic KM System evaluation process consist of selection of the quality dimensions and classify them in to subjective or objective and then applying the hybrid method of using GQM and Score card for data collection. The selected measures which are generated manually or through system way should be stored in any available database and later those will be retrieved for ranking and rating using Weighted Average Mean Method.

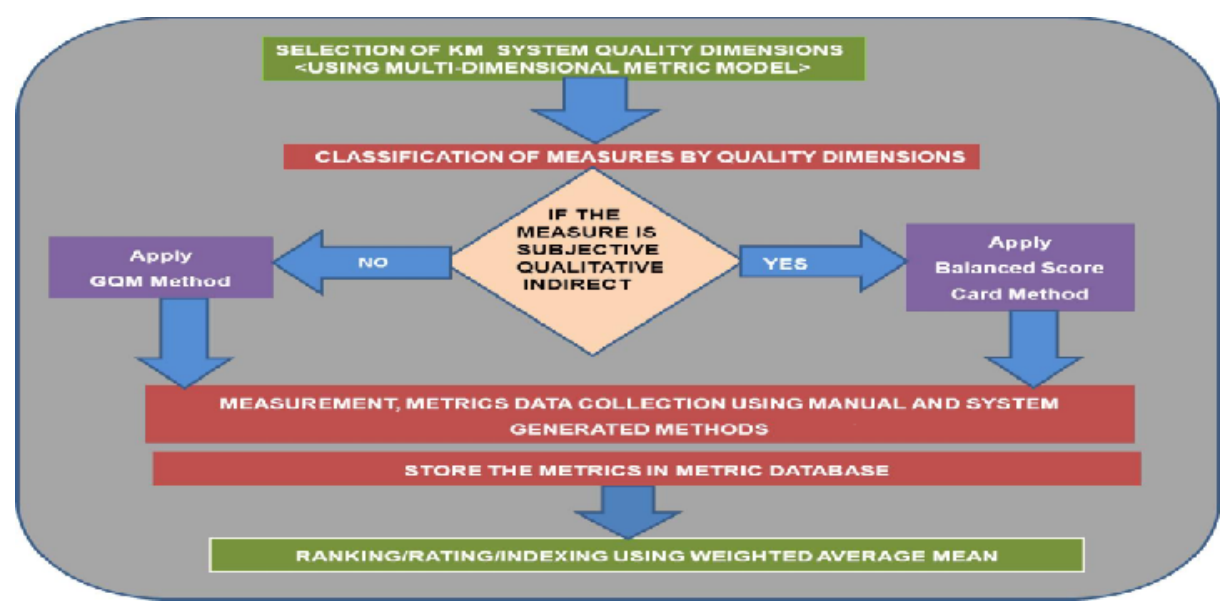

Figure 2: Evaluation Process of KM Systems

\subsection{Quality Factors}

The factors whose effects need to be quantified are called primary quality factors. The features often discussed concerning the overall quality of the knowledge management system are capability, availability, reliability, usability, maintainability and completeness. In the proposed metric model, these quality factors are considered as dimensions. We categorized the factors in two groups such as primary and secondary and evaluators can choose the factors based on the evaluation consideration and outcomes. Continuous Inputs for the KMS Measurement process can be done through standard system programs or tools to monitor the usefulness and responsiveness of supporting technology or the framework or components used for the KMS. The system generated factors may also indicate usability problems and supporting policies for the KMS by introducing the agents which collect these measures.

\subsection{Output Metrics}

Output metrics measure characteristics at the project or task level, such as the effectiveness of lessons-learned for future operations. Direct process output for users provides a picture of the extent to which personnel are drawn to actually using the knowledge system. For a given subject present in the KMS the metric database can be queried and provide the outcomes such as OverallFunctionalityRating, OverallUsabilityRating, OverallAvailabilityRating and OverallEfficiencyRating with respect to the subject or Knowledge Asset available in the KMS/knowledge repository. The output metrics can be calculated or derived based on the 
objective and subjective feedback analysis from the knowledge sharing portal or the whole knowledge infrastructure. Some of these measures can be calculated using system level statistics and also by developing some background agents or web services or through a manual process.

\section{FOUR-DimENSIONAL METRIC MODEL}

As described in the KM System Evaluation process in the earlier section, there are factors and measures which play a critical role for producing the desired outcomes in the KMS evaluation. So, $\mathrm{KM}$ dimensions also called as categories or view-points should be extensively correlated to many factors influencing the results as much as possible. The Knowledge management quality factors enable organizations to strongly indicate what they consider to be important. There are many forces within an organization and Knowledge Management Systems affecting people's learning, sharing, and efficiency, so, it is important to consider the secondary factors as well (Quality Sub Factors). In this section we describe some of the quality dimensions and the respective measures and explain the chosen multi-dimensional metric model [3]. The key is selecting appropriate Quality dimension through factors as represented in the figure (Figure 3) which contains KM quality dimensions such as Functionality, Usability, Availability and Efficiency. The following section discusses about measures and metrics corresponding to some of the prime quality factors which will be given weights in the $80 \%$ category. The diagram below represents the four key dimensions considered for the evaluation of the KM systems. The needed dimensions and attributed can be added as per the evaluation or prediction.

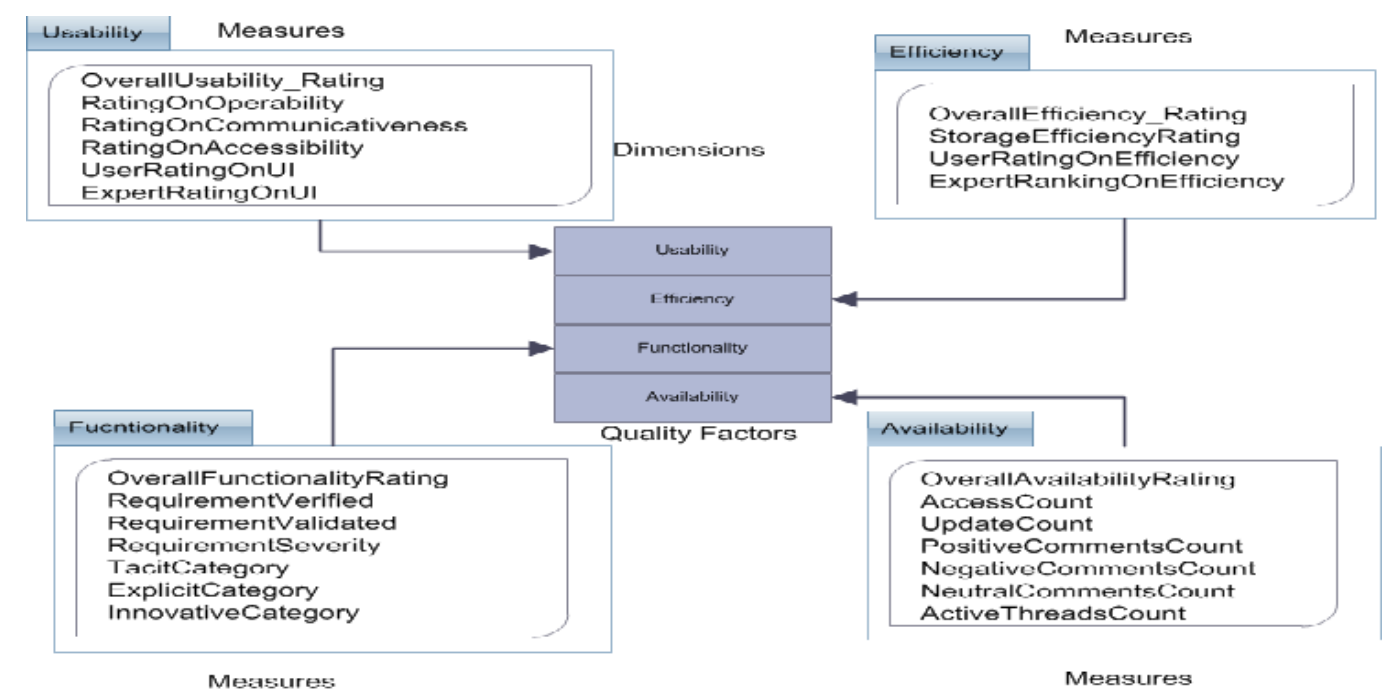

Figure 3: Four-Dimensional KM Metric Model

\subsection{Functionality Measures}

The functionality of KMS can be considered as an entity object in the metric model and its expected behavior will be captured as requirements or attributes of an entity object. 


\subsection{Usability Measures}

The knowledge component should be easily understandable, learnable, and applicable. Usability attribute are the features and characteristics of the software/product/sites that influence the effectiveness, efficiency and satisfaction with which users can achieve specified goals.

\subsection{Availability Measures}

In the context of KMS, Knowledge Availability is whether (or how often) a given knowledge asset is available for use by its intended users.

\subsection{Efficiency Measures}

The knowledge component should state the quickest solution with the least resource requirements.

\subsection{KM Metric Database}

The implementation of metric database is shown in the figure (Figure 4) below which was created to hold the user and expert feedback of the considered dimensions and measures from the database. The database used in the existing infrastructure can be also considered for storing the metrics and measurements. As the volume of data and amount of transactions used for the KM System measurement is very less, there is no need for a dedicated or high performance database and existing database used for infrastructure maintenance or application database can be used to store the schema and data. If the models and databases are systematically developed to be flexible, effective, scalable and surely the data can be explored using mining technique or using WAM Method. The metric database can be created using any industry specific database systems. There are four Key steps in implementing the multi-dimensional metric database:

1. Gather the evaluation factors for assessment.

2. Decide the Quality Factor and Sub Factors

3. Create Data Objects (Tables/XML to hold the Quality Factors and Measures)

4. Upon collecting the measures, store them in the data objects

The metric database for our evaluation experiment, has been implemented using Microsoft Sqlserver 2008 and the data were collected the metrics through Hybrid Approaches [2], manual feeds and also through customized programs. The data got generated based on the inputs from user, expert and system. As you see from the below database ER diagram, our metric database is designed to be flexible to hold any and metric as per the rating from normal user or expert. 


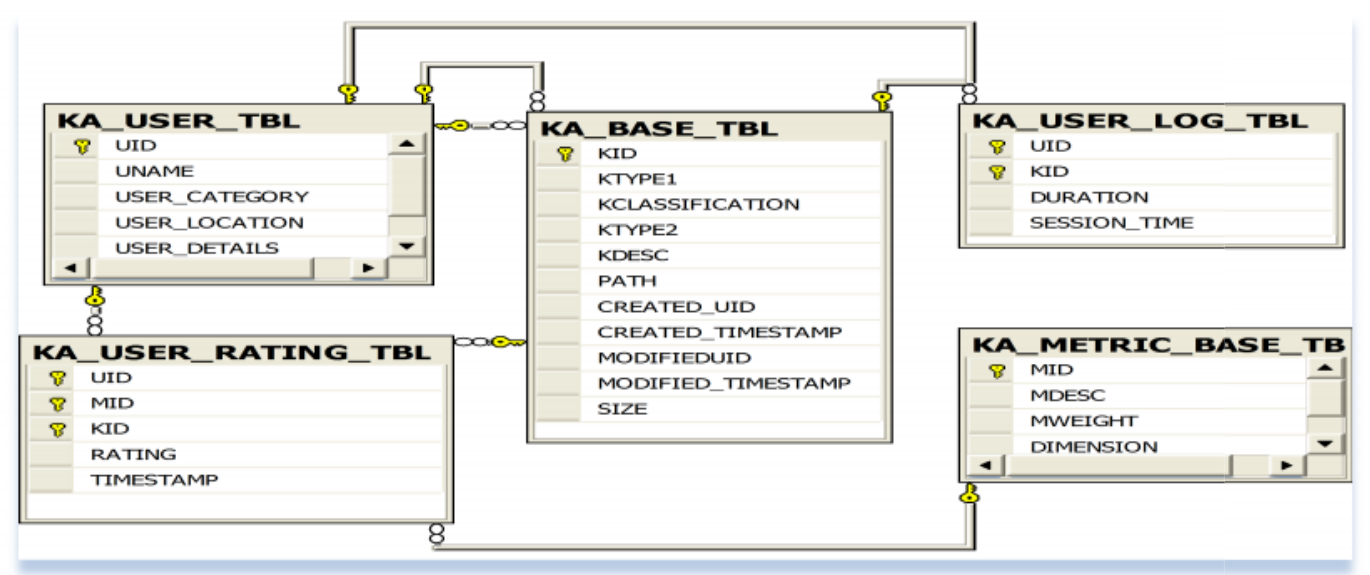

Figure 3: KM Metric Database ER Diagram

The KM Metric database consists of five key tables namely, KA_BASE_TBL which is the base table which contains the knowledge asset created/modified in the knowledge portal or repository. The KA_USER_TBL hold the information about the user name, type and their details. KA_USER_RATING table holds the user feedback on the given measure. The KA_METRIC_BASE_TBL which is our key table which holds the attributes like metric id (MID), Description (MDESC), weight (MWEIGHT) and the corresponding quality factor (DIMENSION).

\section{THE EXPERIMENT - KM SYSTEM ANALYSIS}

The participants were selected from multiple departments of top two engineering colleges with moderate and frequent usage of knowledge portals and knowledge repositories. Initially 360 candidates were selected, but only 162 candidates, with similar profiles, were actually used in the experiment. Prior to conducting the actual experiment, the pilot tests were conducted to validate the approaches and tasks involved. The main aspects of the normal user profiles of the participants used were similar in the following ways:

- Computing knowledge, knowledge of using collaborative tools and basic knowledge in the subject areas present in the KM systems

- Above 20 years of age and below 23 years of age, with English as their learning language for all the subjects

The main aspects of the expert user profiles of the participants used were similar in the following ways:

- Teaching or Training skills; Expert knowledge in the subject area

- Willingness to review and provide feedback of the knowledge assets

- Less than 69 years of age and above 27 years of age with apt qualification

For this experiment, we have considered four important dimensions and allocated evaluation percentage as below:

- $30 \%$ for Functionality; $20 \%$ for Usability

- $20 \%$ for Availability; $20 \%$ for Efficiency

The Table-1 for functionality dimension shows how the evaluation percentage (30\%) is distributed among multiple functionality measures. The third column contains the captured rating which contains aggregated value which is stored in the metric database based on user, expert and 
system feed. The tables 2, 3 and 4 are populated for Usability, Availability and Efficiency Dimension using the aggregated value from the metric database. As mentioned in the earlier sections, for evaluation purpose, we have also considered non-weighted measures and added $10 \%$ to our overall calculation. For an effective and successful KM system, the organization must have multiple support factors such as Environment, Infrastructure, Domain Knowledge and participant's thrust for knowledge sharing. So, we have taken the feedback on these parameters and added to the metric database and populated in the Table 5.

TABLE 1: WEIGHTAGE TABLE FOR FUNCTIONALITY

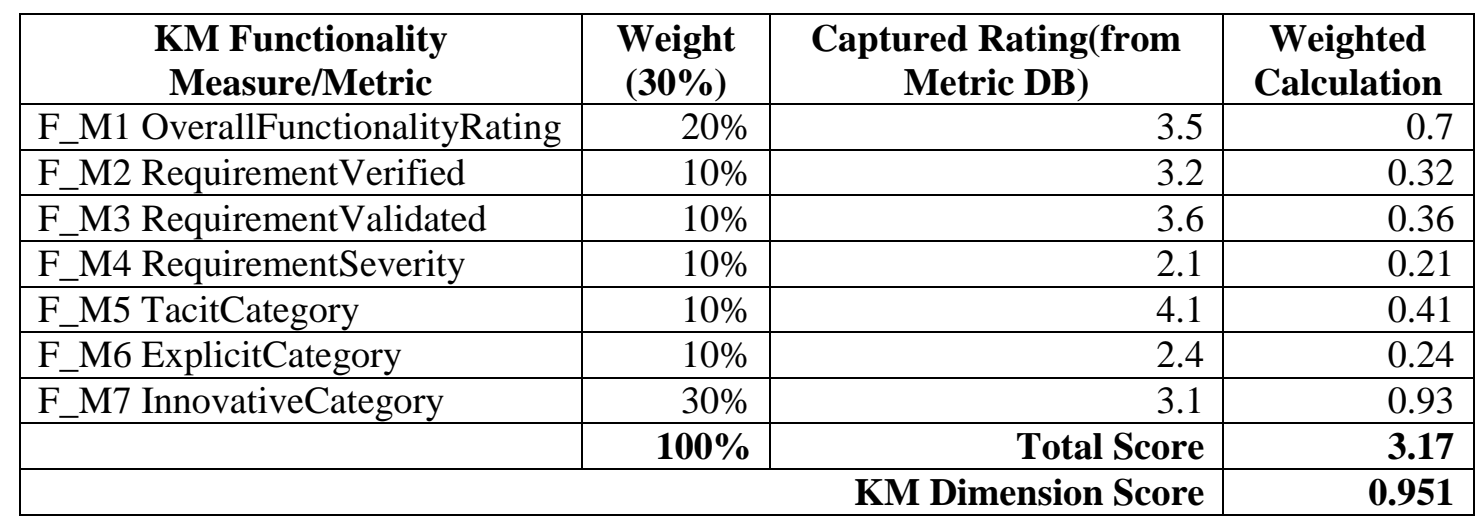

TABLE 2: WEIGHTAGE TABLE FOR USABLITY

\begin{tabular}{|l|r|r|r|}
\hline \multicolumn{1}{|c|}{ KM Usability Measure/Metric } & $\begin{array}{c}\text { Weight } \\
\mathbf{( 2 0 \% )}\end{array}$ & $\begin{array}{l}\text { Captured Rating(from } \\
\text { Metric DB) }\end{array}$ & $\begin{array}{c}\text { Weighted } \\
\text { Calculation }\end{array}$ \\
\hline U_M1 OverallUsability_Rating & $20 \%$ & 3.8 & 0.76 \\
\hline U_M2 RatingOnOperability & $20 \%$ & 4 & 0.8 \\
\hline U_M3 RatingOnCommunicativeness & $10 \%$ & 2.9 & 0.29 \\
\hline U_M4 RatingOnAccessibility & $20 \%$ & 3.5 & 0.7 \\
\hline U_M5 UserRatingOnUI & $20 \%$ & 4 & 0.8 \\
\hline U_M6 ExpertRatingOnUI & $10 \%$ & 3.6 & 0.36 \\
\hline & $\mathbf{1 0 0 \%}$ & Total Score & $\mathbf{3 . 7 1}$ \\
\hline & \multicolumn{3}{|r|}{ KM Dimension Score } \\
\hline
\end{tabular}

TABLE 3. WEIGHTAGE TABLE FOR AVAILABILITY

\begin{tabular}{|c|c|c|c|}
\hline KM Availability Measure/Metric & $\begin{array}{l}\text { Weight } \\
(20 \%)\end{array}$ & $\begin{array}{c}\text { Captured Rating(from } \\
\text { Metric DB) }\end{array}$ & $\begin{array}{l}\text { Weighted } \\
\text { Calculation }\end{array}$ \\
\hline A_M1 OverallAvailablityRating & $20 \%$ & 3.2 & 0.64 \\
\hline A_M2 AccessCount & $10 \%$ & 3.9 & 0.39 \\
\hline A_M3 UpdateCount & $10 \%$ & 3.2 & 0.32 \\
\hline A_M4 PositiveCommentsCount & $10 \%$ & 4.2 & 0.42 \\
\hline A_M5 NegativeCommentsCount & $10 \%$ & 1 & 0.1 \\
\hline A_M6 NeutralCommentsCount & $10 \%$ & 3.7 & 0.37 \\
\hline \multirow[t]{2}{*}{ A_M7 ActiveThreadsCount } & $30 \%$ & 4.1 & 1.23 \\
\hline & $100 \%$ & Total Score & 3.27 \\
\hline \multicolumn{3}{|r|}{ KM Dimension Score } & 0.654 \\
\hline
\end{tabular}


TABLE 4. WEIGHTAGE TABLE FOR EFFICIENCY

\begin{tabular}{|c|c|c|c|}
\hline KM Efficiency Measure/Metric & $\begin{array}{l}\text { Weight } \\
(20 \%)\end{array}$ & $\begin{array}{c}\text { Captured Rating(from } \\
\text { Metric DB) }\end{array}$ & $\begin{array}{c}\text { Weighted } \\
\text { Calculation }\end{array}$ \\
\hline E_M1 OverallEfficencyRating & $20 \%$ & 3.9 & 0.78 \\
\hline E_M2 StorageEfficiencyRating & $20 \%$ & 3.5 & 0.7 \\
\hline E_M3 UserRatingOnEfficiency & $30 \%$ & 4.1 & 1.23 \\
\hline \multirow{2}{*}{ E_M4 ExpertRankingOnEfficiency } & $30 \%$ & 3.6 & 1.08 \\
\hline & $100 \%$ & Total Score & 3.79 \\
\hline & & KM Dimension Score & 0.758 \\
\hline
\end{tabular}

TABLE 5. NON-WEIGHTED MEASURES TABLE

\begin{tabular}{|l|r|}
\hline \multicolumn{1}{|c|}{ KM Non Weighted Measure/Metric (10\%) } & Captured Rating(from Metric DB) \\
\hline NW_M1Supporting KM Infrastructure & 3 \\
\hline NW_M2 Management Support & 2 \\
\hline NW_M3 Condusive Environment & 2 \\
\hline NW_M4 Particpant's Subject Knowledge & 2 \\
\hline NW_M5 Participants Thrust for Knowledge & 3 \\
Collabration & 11 \\
\hline Total Non Weight Value & $\mathbf{2 . 2}$ \\
\hline Average & $\mathbf{0 . 2 2}$ \\
\hline 10\% of Non Weighted Dimension Score & \\
\hline
\end{tabular}

TABLE 6. OVERALL EFFECTIVENESS TABLE

\begin{tabular}{|l|l|c|}
\hline Allocation & \multicolumn{1}{|c|}{ Type } & \multicolumn{1}{c|}{ Derived Score } \\
\hline $90 \%$ & $\begin{array}{l}\text { Weighted Dimension } \\
\text { Group }\end{array}$ & 2.79 \\
\hline $10 \%$ & $\begin{array}{l}\text { Non Weighted } \\
\text { Dimension Group }\end{array}$ & 0.22 \\
\hline \multicolumn{2}{|c|}{ Overall KM System Score } & 3.01 \\
\hline
\end{tabular}

TABLE 7. RANKING AND RATING TABLE

\begin{tabular}{|l|l|r|}
\hline Rank & \multicolumn{1}{|c|}{ Category } & Rating \\
\hline 1 & Outstanding & 5 \\
\hline 2 & Extremely Effective & 4 \\
\hline 3 & Effective & 2 \\
\hline 4 & Below Effective & 1 \\
\hline 5 & Not Effective & \\
\hline
\end{tabular}

The Table 6 is the summary table and contains both weighted and non-weighted dimension group. The weighted dimension group score is 2.79 , which has been derived by adding all the four quality dimension scores. The non-weighted dimension group was given 0.22 and both derived scores were added to make up overall KM System Effectiveness Score. The KM system was evaluated by the users and experts with the score 3.01. The data obtained for this experiment concerned with the effectiveness of the KM system of the engineering subjects/knowledge assets. 


\section{RESTFUl RESOURCES}

The term REST comes from Roy Fielding's PhD dissertation, published in 2000, and it stands for REpresentational State Transfer. REST by itself is not an architecture; REST is a set of constraints that, when applied to the design of a system, creates a software architectural style. A RESTful resource is anything that is addressable over the Web. The resource can be accessed and transferred between clients and servers and it is a logical, temporal mapping to a concept in the problem domain for which we are implementing a solution. Because we are using HTTP to communicate, we can transfer any kind of information that can be passed between clients and servers. A Uniform Resource Identifier, or URI, in a RESTful web service is a hyperlink to a resource, and it's the only means for clients and servers to exchange representations.

In modern web application development we limit design and implementation ambiguity, because we have four specific actions that we can take upon resources-Create, Retrieve, Update, and Delete (CRUD). Therefore, with the delineated roles for resources and representations, we can now map our CRUD actions to the HTTP methods POST, GET, PUT, and DELETE. The method GET is used to RETRIEVE resources. We need to determine what a resource is in the context of our web service and what type of representation we're exchanging.

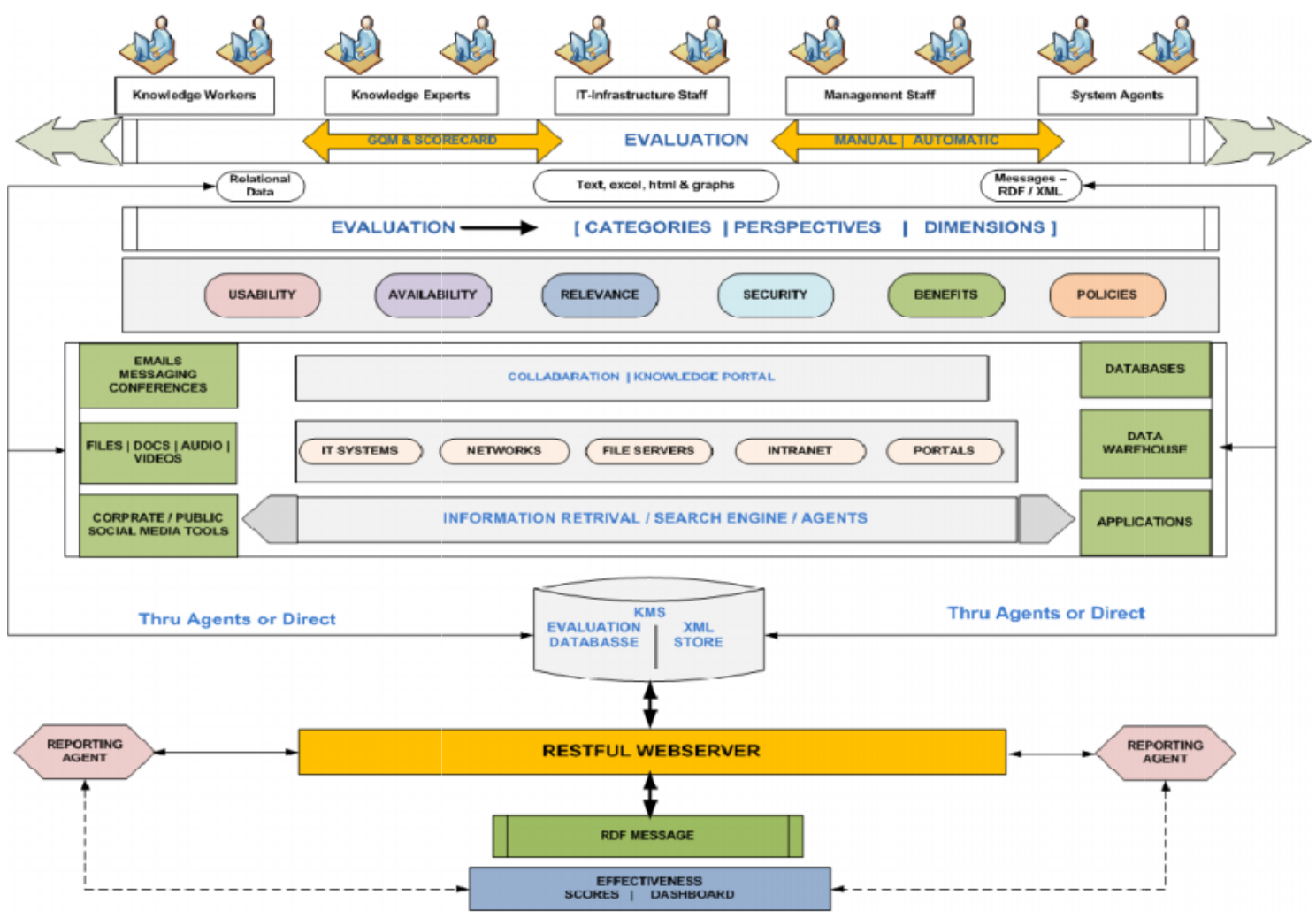

Figure 5: KM System Evaluation Framework using RESTful Architecture

The above framework has been redefined based on our KMS technology Framework [2] found in Figure-1, to adapt RESTful Architecture to evaluate any KM System using RESTful resources and Web services. The metrics based on the feedback from Knowledge Workers, Experts, IT 
Infrastructure Staff, management staff and also from the System Agents can be stored in the KMS Metric/Evaluation database or in the form of XML. The RESTFul Webserver can be designed in such a way, to retrieve the metrics from the KM Metric database and produce the results in the form of RDF Message.

The Reporting Agent can be used to transform the metric database through RESTful server to represent in a Text or Graphical or XML form. The agents can be developed to feed the data through messages to RESTful Web server first and then it can be stored in a traditional metric database. In order to handle the KM System evaluation, we need to construct a web service first. The XML representation of the key dimensions from the four dimensional model is given below:

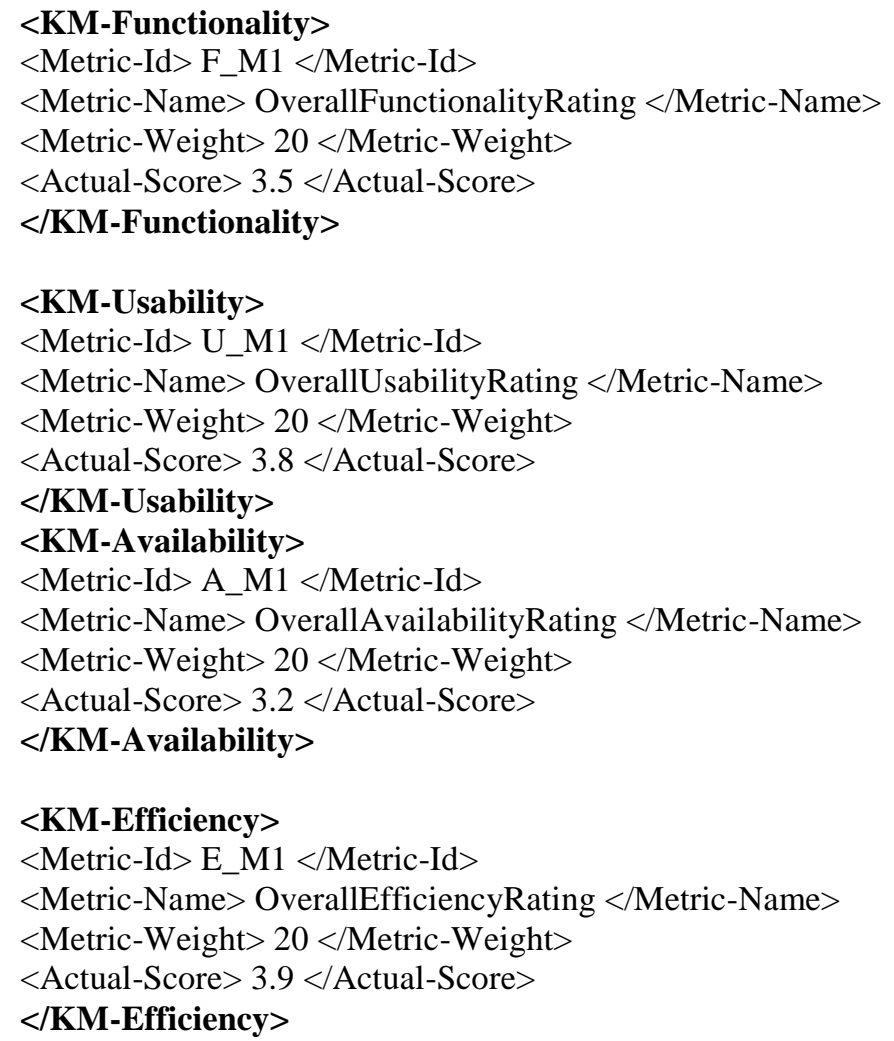

With the representations defined, we now assume URIs of the form http://KM-SystemEval.com/KM-Functionality to access a list of functionality metrics, and http://KM-SystemEval.com/KM-Functionality/\{ Metric-Id \} to access a Metric that has the unique identifier of value Metric-Id. For instance, if we wanted the retrieve or record for a metric with the metric id U_M1, we make a request to the URI http://KM-System-Eval.com/KM-Functionality/U_M1.

The method GET is used to RETRIEVE resources, the method POST is used to CREATE resources, the method PUT is used to UPDATE resources and the method DELETE is used to DELETE representations. The table 8 contains the URI and HTTP verb mapping for business operations of the KM System Evaluation. 
TABLE 8: URI-HTTP MAPPING TABLE

\begin{tabular}{|c|r|r|r|r|}
\hline URI & $\begin{array}{c}\text { HTTP } \\
\text { Method }\end{array}$ & COLLECTION & OPERATION & EVALUATION OPERATION \\
\hline /F_M1 & GET & /KM-Functionality & RETRIEVE & GET SCORES \\
\hline /F_M1 & POST & /KM-Functionality & CREATE & STORE SCORES \\
\hline /U_M1 & GET & /KM-Usability & RETRIEVE & GET SCORES \\
\hline /U_M1 & POST & /KM-Usability & CREATE & STORE SCORES \\
\hline / A_M1 & GET & /KM-Availability & RETRIEVE & GET SCORES \\
\hline /A_M1 & POST & /KM-Availability & CREATE & STORE SCORES \\
\hline /E_M1 & GET & /KM-Relevance & RETRIEVE & GET SCORES \\
\hline /E_M1 & POST & /KM-Relevance & CREATE & STORE SCORES \\
\hline
\end{tabular}

Designing RESTful web services is not different from designing traditional web applications. We can think of RESTful web service design as being similar to Object Oriented Design (OOD).

The underlying RESTful web service design principles can be summarized in the following four steps:

1. Requirements gathering for KM System Evaluation

2. Resource identification based on proposed multi-dimensional model

3. Resource representation definition-between clients (User Interfaces or Agents) and servers (KM metric database or Web Server). For KM metrics, we can use XHTML.

4. URI definition - with resources in place, we need to define the API, which consists of URIs for clients and servers to exchange resources' representations.

\section{CONCLUSION}

In this research work, we have attempted to build a four dimensional model, the metric database and a proven statistical technique Weighted Average Mean to validate the effectiveness of the knowledge sharing in the KM systems. By referring to Table 6 and the guideline Table 7 for ranking and rating, it is clear, that the evaluated $\mathrm{KM}$ system is effective as the KM system got the overall KM System Score of 3.01. The results obtained through the experiment, proves that the combination of Metric Database with a Statistical technique such as WAM could be useful to predict the usefulness and effectiveness of the KM Systems. In complex KM Systems, the KM System Infrastructure is heterogeneous and contains multiple social and economic factors [2] and hence more dimensions have to be considered. Automation would be flexible and cost effective using RESTful Resources and Web Services when used in conjunction with the metric database for better metrics collection and reporting.

\section{REFERENCES}

[1] Dayanandan Venkata Subramanian, Angelina Geetha (2011), "Guidelines and Effectiveness of Using Social Media Tools For Knowledge Sharing Communities”, National Conference on Knowledge Intelligence and Telematics, Gujarat, India

[2] Venkata S Dayanandan, Angelina Geeteha(2011), “Adaptation of Goal Question Metric Technique For Evaluation of Knowledge Management Systems", Review of Knowledge Management Journal, Volume 1, Jan-June 2011, ISSN:2231-5845 
[3] Venkata S Dayanandan, Angelina Geeteha, Mohammed Hussain(2011), "Measurement Process and Multi-dimensional Model For Evaluating Knowledge Management Systems", International Conference on Research and Innovation in Information Systems, International Islamic University, Malaysia (IIUM) and Universiti Teknologi Malaysia(UTM), Indexed by IEEE \& SCOPUS, November 2011, IEEE ISBN: 978-1-61284-295-0

[4] Diwakar Krishnamurthy, Jerry Rolia, and Min Xu1(2011), "WAM - The Weighted Average Method for Predicting the Performance of Systems with Bursts of Customer Sessions, University of Calgary, Calgary, AB, Canada, IEEE TRANSACTIONS ON SOFTWARE ENGINEERING, 0098-5589/11

[5] Jiawei Han, Yizhou Sun, Xifeng Yan, Philip S. Yu(2010), "Mining Knowledge from Databases: An Information Network Analysis Approach", SIGMOD'10, June 6-11, 2010, Indianapolis, Indiana, USA. ACM 978-1-4503-0032-2/10/06.

[6] Behrang ZadJabbari, Pornpit Wongthongtham, Farookh Khadeer Hussain(2010), “Ontology based Approach in Knowledge Sharing Measurement", Communications of the IBIMA Volume 11", 2009 ISSN: 1943-7765

[7] Ashleigh-Jane Thompson, Elizabeth A Kemp, "Web 2.0: Extending the framework for heuristic evaluation", Proceedings of the 10th international conference NZ Chaperte of the ACM's special interest group on Human Computer Interface, 2009

[8] Stephanie D Swindler, Christian E Randall, Gary S Beisner II, "An application of heuristic techniques for evaluation of collaborative systems interfaces", nternational Symposium on Collabarative Technologies and systems, 2006.

[9] A.Kankanhalli and B.C.Y. Tan, National University Of Singapore, "K.M Metrics : A Review and Directions for future research", Proceedings of the 37th Annual Hawaii International Conference in Knowledge Management, 2004

[10] B. Martens and W. Jabi, "Knowledge Portal as a new paradigm for scientific publishing and collaboration", IT conference, 2004 